\title{
Evaluación del programa de computación educativo para la aplicación de escalas ISS y AIS en el servicio de urgencias para residentes de ortopedia de la Secretaría de Salud de la Ciudad de México
}

\author{
Evaluation of educational computer program for applying ISS and AIS scales in the \\ emergency room for Orthopedic Residents of the Secretary of Health from Mexico City
}

Muñoz-Morales O, * Martínez-Arredondo H, ${ }^{\ddagger}$ Franco-Valencia M, ${ }^{\S}$ Urquijo-Morales A ${ }^{\|}$

Hospital General Xoco.

RESUMEN. Introducción: Se creó el programa HAISS (Herramienta de Aprendizaje ISS) para mejorar la factibilidad y aprendizaje de las escalas de gravedad en trauma AIS (Abbreviated Injury Score) e ISS (Injury Severity Score). Objetivo: Obtener una herramienta de computación que promueva el aprendizaje y facilite el uso adecuado de las escalas AIS e ISS. Material y métodos: Se toma una muestra de 40 residentes de primero a cuarto grado de ortopedia de cuatro hospitales de la Secretaría de Salud. Se realizó un estudio longitudinal prospectivo con estadística analítica. Se entregaron 10 casos clínicos para obtención de ISS mediante la escala AIS, se utilizó el manual AIS 2005 actualización 2008, se plantearon dos preguntas para evaluar facilidad de uso y factibilidad, se midió el tiempo de realización. Se repitió el proceso al mes con el programa HAISS. Resultados: Con ayuda del programa HAISS se redujo el tiempo para resolver los 10 casos en más de $50 \%$, la aceptación por parte del usuario de la escala AIS también mejoró. La fiabilidad de obtener la codificación de las lesiones con AIS no mejoró, pero no se ve afectada la obtención del ISS. Conclusiones: El programa HAISS mostró aceptación en un grupo de residentes de ortopedia, mejoró en forma subjetiva su uso y además ocupa poco tiempo.

Palabras clave: Informática médica, software, educación, índice de severidad de lesiones, escala abreviada de lesiones.
ABSTRACT. Introduction: The HAISS (Herramienta de Aprendizaje ISS) program was created to enhance the feasibility and learning of trauma severity scores AIS (Abbreviated Injury Score) and ISS (Injury Severity Score). Objective: Get a computer tool that promotes learning and facilitate proper use of AIS and ISS. Material and methods: A sample of 40 orthopedic residents from 1st to 4th grade from 4 hospitals of the Ministry of Health was taken. A prospective longitudinal study was conducted with analytical statistics. 10 clinical cases for obtaining ISS by AIS score were delivered, this was done with the AIS Manual 2005 update 2008, two questions were conducted to evaluate the feasibility and ease of use, the time to perform was measured. The process was repeated a month later with the HAISS program. Results: Using the HAISS program reduced the time to resolve the 10 cases by more than $50 \%$, acceptance by the user of the AIS scale also improved. Reliability to obtain the codification of lesions with AIS did not improve, but it did not affected ISS. Conclusions: The HAISS computer program is useful for orthopedic residents to accept the AIS score and ISS score mainly because it improved the ease of use and makes it more user-friendly, it also takes less time to use it.

Keywords: Medical informatics, software, education, injury severity score, abbreviated injury scale.

\section{Nivel de evidencia: IV}

* Médico adscrito Hospital General de Zona Núm. 29, IMSS.

${ }^{\ddagger}$ Médico adscrito del Servicio de Ortopedia Hospital General La Villa.

${ }^{\S}$ Médico adscrito del Servicio de Ortopedia Hospital General Xoco.

" Ingeniero en Sistemas de Computación.

Dirección para correspondencia:

Omar Muñoz Morales

Calle F, Manzana II, Núm. 4, Col. Educación, C.P. 0400, Alcaldía Coyoacán, Ciudad de México. Tel: 55442076.

E-mail: ommk2000@hotmail.com

Este artículo puede ser consultado en versión completa en: www.medigraphic.com/actaortopedica 


\section{Introducción}

La computación en el área médica ha evolucionado de ser un procesador de datos a una herramienta en diferentes áreas como la administrativa, la investigación y la académica. En esta última se han desarrollado desde juegos hasta simuladores con la meta de mejorar el aprendizaje. Es en este punto donde adquiere importancia la creación de programas de cómputo que faciliten el aprendizaje así como la aceptación por parte de usuarios de sistemas de clasificación o manejo de información cuyo uso, de otra forma, no sería viable en la práctica diaria. ${ }^{1}$ Algunos ejemplos de la informática médica en el aprendizaje son el empleo de carpetas de estudio digitales basadas en web 2.0 WordPress, utilizadas por algunas instituciones donde el alumno tiene contacto directo con su tutor obteniendo una retroalimentación constante. Existen programas como el Web Quest, el cual trata de inducir al estudiante al autoaprendizaje mediante casos y preguntas en línea y de esta manera trata de orientar a un aprendizaje autónomo así como a familiarizarse con la gran cantidad de lugares de donde se puede obtener información con una base critica. ${ }^{2,3,4,5}$ Otro método ampliamente utilizado es la didáctica con ayuda de computación, mediante juegos se ha demostrado que se puede estimular el aprendizaje. ${ }^{6}$ Esto es aplicable a campos tan áridos como las ciencias básicas o basadas en imagen como la radiología. ${ }^{7}$

Un punto a tomar en cuenta es la simulación como método de enseñanza debido a que cada vez toma más fuerza, aunque aún no se cuenta con estudios suficientes con calidad para validar su efectividad, es una rama de la informática médica que parece prometedora ${ }^{8,9}$ La informática médica adquiere relevancia en la actualidad para complementar el aprendizaje del estudiante de medicina así como para facilitar el mismo.

Los métodos digitales para el aprendizaje son más accesibles. Hace unos años el uso de programas de cómputo para aprender medicina estaba limitado a la PC y las laptops. Desde 2009 ha habido un gran aumento en la compra de tecnología de información en el sector salud sobre todo en dispositivos móviles. ${ }^{10}$ En un estudio de 2011 se encontró que $85 \%$ de la población estudiantil tenía acceso a un dispositivo móvil, ${ }^{11}$ surgió un debate de si es la forma adecuada para aprender, las razones a favor fueron su facilidad de acceso, variedad de costos, aprendizaje en el momento y mejor comunicación estudiante tutor, por otro lado su principal desventaja es que son tecnología dependientes. ${ }^{12} \mathrm{Al}$ final, la conclusión es que $50 \%$ de los estudiantes de medicina ocupan aplicaciones o programas para aprender; sin embargo, hay una discrepancia entre sus ganas de aprender por medio de la informática médica y lo que en realidad aprovechan del programa; no obstante, esto último puede ser solventado con estimulación por parte de su institución o tutor. Es importante la creación de herramientas que faciliten el aprendizaje, por lo que en este caso se eligió un programa de cómputo que facilite el uso y comprensión de la escala AIS 2005 actualización 2008 (Abbreviated Injury Score) para obtener ISS (Injury Severity Score) mejorando de esta misma forma la aceptación por parte del usuario.

La escala AIS se define como un sistema de codificación de la gravedad global de las lesiones traumáticas, consensuado y basado en la anatomía corporal, que clasifica cada lesión según la región del cuerpo de acuerdo con su importancia relativa en una escala ordinal de seis puntos, es una escala adecuada para la predicción de gravedad de lesiones. El ISS es un predictor de mortalidad, el cual asigna a las lesiones presentadas por un paciente una puntuación basada en AIS, a continuación se toman las tres lesiones con puntaje más alto, después cada una se eleva al cuadrado y se suman todas, con lo que se obtienen puntuaciones que van de 0 a 75 , un puntaje arriba de 17 se considera mal pronóstico.

El programa de cómputo diseñado para este estudio, llamado herramienta aprendizaje ISS (HAISS), se creó con ayuda de MySQL y Java, la función principal del software es obtener una herramienta que nos ayude a enseñar a médicos residentes de ortopedia a usar la escala AIS para obtener ISS con poca variabilidad interobservador e intraobservador, con mejor factibilidad de uso y una aceptación por parte del usuario para de esta manera poder usarse en nuestro medio. Se diseñó para utilizarse por Windows, Linux y smartphones Android, el programa funciona con un servidor central y por medio de Internet se tiene acceso a la base de datos, por lo que no se requiere tener instalado el programa en sus equipos. El programa digitaliza el manual de las escala AIS 2005 actualización 2008 y lo organiza en ventanas, asimismo incluye un instructivo para utilizar la escala AIS e ISS.

\section{Material y métodos}

Estudio descriptivo, longitudinal y prospectivo, con una muestra por conveniencia de 40 médicos residentes de ortopedia de la Secretaría de Salud. Se tomaron en cuenta variables como edad, sexo y hospital sede.

Para el análisis estadístico se utilizaron medidas de tendencia central así como estadística analítica como kappa para variables categóricas y prueba de Wilcoxon.

Se utilizó una hoja de recolección de datos, la cual cuenta con preguntas de factibilidad, casos clínicos, edad, tiempo, grado académico, ficha de identificación, autorización y firma de consentimiento informado.

Se trata de un estudio previo y posterior de una intervención donde se evalúa el uso de la escala AIS para obtener ISS con el manual AIS 2005 actualización 2008 y con el programa de computación HAISS. Se realizaron dos mediciones del tiempo para completar las evaluaciones de los 10 casos con un mes de separación para eliminar sesgo de memoria, la primera medición se realizó con el manual de la escala y la segunda medición con el programa HAISS. Cada medición se aplica a 40 residentes de cuatro hospitales, la cual consta de 10 casos clínicos, se pidió el AIS de cada caso para después obtener el ISS, con esto se evalúa la variabilidad intraobservador e interobservador. 
Las preguntas planteadas al final con cuatro respuestas posibles fueron: pegunta $A$ : ¿considera que la escala es de fácil uso? y la pregunta $\mathrm{B}$ : ¿considera factible usar la escala en un día laboral normal? Con ello esperamos evaluar la utilidad y facilidad del uso del programa.

Consideraciones éticas: el protocolo de estudio y toda la investigación en general fue puesta a evaluación del Comité de Ética de Hospital General Xoco siendo aceptado por el mismo. La hoja de recolección de datos cuenta con una sección de consentimiento informado, el cual firmaron todos los participantes. Cabe destacar que previo al estudio se informó a los participantes en qué consistía el estudio y su finalidad.

\section{Resultados}

Los tiempos de respuesta para la primera y la segunda medición se muestran en las Figuras 1 y 2; se encontró que en algunos años el tiempo para la obtención de ISS se redujo hasta en $50 \%$.

En cuanto a las dos preguntas de facilidad de uso: la pregunta A en la primera medición reporta que sólo 2.1\% considera la escala de fácil uso y el resto se reparte de manera equitativa entre casi siempre, ocasionalmente y nunca; en la segunda pregunta sólo $6.3 \%$ lo considera siempre, casi siempre $4.2 \%$, ocasionalmente $25 \%$ y nunca $47 \%$.

En la segunda medición se notaron cambios, en la pregunta A $27.1 \%$ siempre, $41.7 \%$ casi siempre y $12.5 \%$ y $2.1 \%$ ocasionalmente o nunca respectivamente; en la pregunta B se encuentra siempre $22.9 \%$, casi siempre $25.0 \%$, ocasionalmente $31.3 \%$ y nunca $4.2 \%$, con lo cual inferimos que mejora la aceptación del usuario a la obtención del ISS con AIS 2005 actualización 2008.

En cuanto a la concordancia entre lo obtenido en AIS y el valor de referencia de ISS se aprecia que en la primera medición se obtiene un kappa de 0.221 y en la segunda medición se observa un kappa de 0.254 , por lo tanto las dos se encuentran en una concordancia aceptable. Se intentó

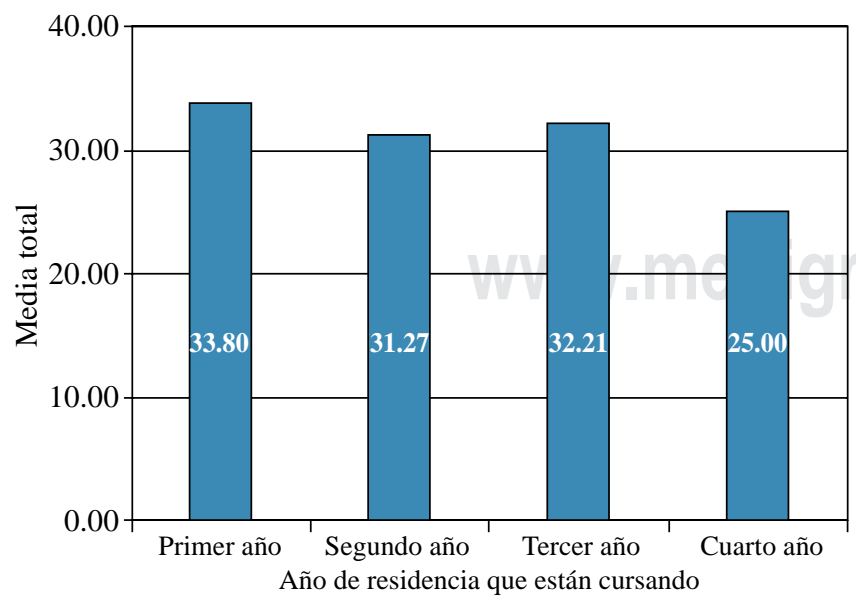

Figura 1: Media de tiempo en minutos por año de residencia primera medición.

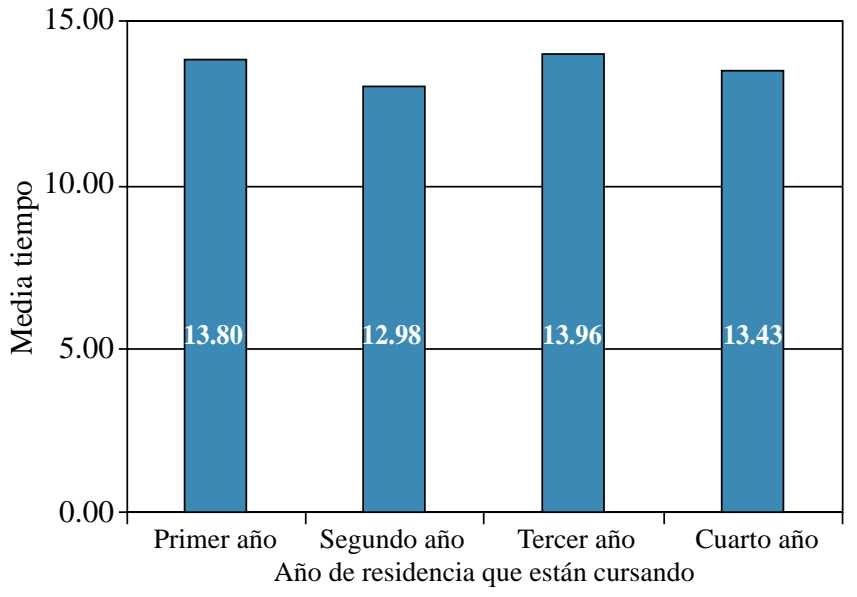

Figura 2: Media de tiempo en minutos por año de residencia segunda medición.

realizar pruebas de fiabilidad (correlación interobservador y ANOVA) para evaluar lo obtenido por el observador y el valor de referencia; sin embargo, como prácticamente no hubo diferencias, se obtuvieron errores tanto en la primera medición como en la segunda, por lo que se ocupa la prueba de Wilcoxon de los rangos con signo de muestras relacionas que recomienda mantener hipótesis nula. Siendo la hipótesis nula, aunque el AIS no es el mismo en todos los casos, el ISS no se modifica.

\section{Discusión}

No hay registros en nuestro país sobre transformar un manual de las dimensiones de la escala AIS 2005 actualización 2008 en un programa que ayude a mejorar tres puntos: 1. La aceptación por parte del usuario, 2. El tiempo para obtener el AIS y 3. En aprender el uso de la escala. Tampoco hay estudios que nos hablen acerca del uso de esta escala.

Otro punto importante de este programa tanto en educación como en aplicación a la clínica es debido a que se puede ocupar para otras escalas o servir como base para crear otras herramientas que ayuden en las mismas áreas en que HAISS mejoró.

Es importante mencionar que no se necesitan grandes recursos para lograr realizar un programa de cómputo que ayude al aprendizaje, un ejemplo de esto es el portafolio en WordPress realizado por Javier Aviela y colaboradores en el que se usan programas de fuente abierta como MySQL para bases de datos y servidor Apache, los cuales no requieren una inversión como tal obteniendo buenos resultados e invitando al uso de fuentes abiertas de programas; como herramientas de aprendizaje nosotros usamos MySQL y Java. Zohreh Badiyepeymaie Jahromi utiliza el Web Quest como método de autoaprendizaje, pero menciona que a pesar de que es un buen método no es útil para todos los estudiantes y que puede incluso ocasionar estrés y frustración en el usuario, considerando esto se debe tomar en cuenta que HAISS puede no ser $100 \%$ útil para todos los usuarios 
siendo la mejor opción un sistema flexible de aprendizaje donde el HAISS sólo sea una herramienta complementaria de aprendizaje, algo similar se observa en el estudio de Annemarie M. den Harder en el que se analiza la interacción entre computadora y humano en el aprendizaje de radiología y llegan a la conclusión de que la computación puede ser útil en el aprendizaje; sin embargo, no todos los usuarios la aceptan como tal. Ya se comentó sobre la utilidad de programas abiertos así como de la utilidad de los programas de cómputo en el aprendizaje en otros estudios; no obstante, queda un punto importante a evaluar que es la aceptación y uso de nuevas tecnologías por parte de los posibles usuarios, Si Fan comenta que gran parte de la población ya tiene acceso a tecnologías que pueden permitir el aprendizaje; sin embargo, de las 22 herramientas de aprendizaje que analizaron en su estudio, se encontró que menos de la mitad eran usadas cotidianamente en comparación con el presente artículo, en el que se observó una mejoría apreciable en la aceptación del uso de la escala, aunque falta comprobar si su uso a largo plazo se mantendrá con la misma aceptación y hacia una población mayor como todo el personal de nuestro servicio de urgencias.

Una limitación del estudio y área a explorar es el uso del programa HAISS en médicos adscritos así como de otros servicios que se encuentran en constante contacto con trauma como urgencias o cirugía general, entre otros. Otra posibilidad sería poder extender el estudio a otras instituciones de salud como el Instituto Mexicano del Seguro Social o el Instituto de Seguridad y Servicios Sociales de los Trabajadores del Estado para observar si funciona de la misma manera.

Por otro lado, cabe señalar que aún queda campo de investigación por parte de la escala, ya que además de ser un buen predictor de mortalidad es una escala codificada con números en la que cada lesión tiene un código, lo que podría ayudar a la creación de una base de datos para lograr hacer una adecuada epidemiologia de los hospitales de la Secretaría de Salud, debido a que en realidad no existe en la actualidad una base de datos confiable para tener una epidemiología adecuada de nuestro sistema de salud, con la posibilidad de obtener información como ficha de identificación del paciente, diagnóstico, tratamiento y resultado. $Y$ un punto a tomar en cuenta y no dejar de lado son los simuladores, ya que el uso de esta escala como de otras herramientas de la informática médica con simuladores sofisticados podría ayudar a un mejor desenvolvimiento en la clínica.

En la actualidad el acceso a los medios digitales de información es cada vez más sencillo, por lo que se tiene que evolucionar en la aplicación de nuevas tecnologías como la informática médica para lograr motivar a médicos al uso y aprendizaje de herramientas que les pueden ser útiles en su vida diaria.

Las escalas AIS e ISS son útiles en nuestra institución y se puede obtener mucha información de éstas, por lo que se recomienda antes de promover su uso generalizado un estudio más amplio, mejores controles de selección y análisis para considerar su aplicación en el sector salud nacional.
Otra recomendación es que al introducir herramientas nuevas o poco usadas nos acordemos de que nos encontramos en una época en la que la informática médica puede ser una gran herramienta para un mejor aprendizaje, optimizar tiempo y recursos.

\section{Conclusiones}

El programa de cómputo HAISS mejoró el tiempo de clasificación así como la aceptación de los residentes de ortopedia para la obtención del AIS. No mejoró la relación interobservador para obtener el código AIS, aunque cabe señalar que al final no altera significativamente la obtención del ISS.

Este programa es una herramienta para facilitar el uso y lograr una mejor aceptación por parte de los residentes de las escalas AIS e ISS.

Bibliografía

1. http://www.facmed.unam.mx/emc/computo/infomedic/historia.htm

2. Avila J, Sostmann K, Breckwoldt J, Peters H. Evaluation of the free, open source software WordPress as electronic portfolio system in undergraduate medical education. BMC Med Educ. 2016; 16(1): 157.

3. Badiyepeymaie Jahromi Z, Mosalanejad L, Rezaee R. The effect of web quest and team-based learning on students' self-regulation. $J$ Adv Med Educ Prof. 2016; 4(2): 80-7.

4. Cerutti B, Blondon K, Galetto A. Long-menu questions in computerbased assessments: a retrospective observational study. BMC Med Educ. 2016; 16(1): 55.

5. Gharib M, Zolfaghari M, Mojtahedzadeh R, Mohammadi A, Gharib A. Promotion of critical thinking in e-learning: a qualitative study on the experiences of instructors and students. Adv Med Educ Pract. 2016; 7: 271.

6. Del Cura-González I, López-Rodríguez JA, Sanz-Cuesta T, Rodríguez-Barrientos R, Martín-Fernández J, Ariza-Cardiel G, et al. Effectiveness of a strategy that uses educational games to implement clinical practice guidelines among Spanish residents of family and community medicine (e-EDUCAGUIA project): a clinical trial by clusters. Implement Sci. 2015; 11(1): 71.

7. Den Harder AM, Frijlingh M, Ravesloot CJ, Oosterbaan AE, van der Gijp A. The importance of human-computer interaction in radiology e-learning. J Digit Imaging. 2016; 29(2): 195-205.

8. Gordon CJ, Ryall T, Judd B. Simulation-based assessments in health professional education: a systematic review. J Multidiscip Healthc. 2016; 9: 69.

9. Makransky G, Bonde MT, Wulff JSG, Wandall J, Hood M, Creed $\mathrm{PA}$, et al. Simulation based virtual learning environment in medical genetics counseling: an example of bridging the gap between theory and practice in medical education. BMC Med Educ. 2016; 16(1): 98.

10. Masic I, Begic E, Begic N. Use and knowledge on the information technologies in medical education -Bosnian and Herzegovinian experience. Mater Socio Medica. 2016; 28(2): 84.

11. Fan S, Radford J, Fabian D. A mixed-method research to investigate the adoption of mobile devices and Web2.0 technologies among medical students and educators. BMC Med Inform Decis Mak. 2016; 16(1): 43.

12. Walsh K. Mobile learning in medical education: review. Ethiop $J$ Health Sci. 2015; 25(4): 363-6.

Financiamiento: Ninguno.

Conflicto de intereses: Los autores declaran que no tienen conflicto de intereses.

Presentaciones previas: Ninguna. 